\title{
Simulation of NURBS Curve Interpolation Algorithm and Five S-phased Acceleration and Deceleration Control Algorithm
}

\author{
Gengzhu Wang \\ University of Science and Technology Liaoning, Anshan, Liaoning, 114051, China \\ wgz20203@sina.com
}

Keywords: Simulation, Interpolation algorithm, Acceleration and deceleration control

\begin{abstract}
The computer simulation method is used to test the correctness of the NURBS curve interpolation algorithm, through the comparison of the five S-phased curve acceleration and deceleration control method and the line acceleration and deceleration control method, to validate the superiority of the five S-phased curve acceleration and deceleration control method.
\end{abstract}

\section{Introduction}

At present the majority of $\mathrm{CNC}$ lathes only have the straight line and the circular arc interpolation functions. When carrying on the production of free curve surface (for example molds, airplane wings), the off-line cutting tool path has to be generated. Using massive small straight lines replaces the original complex curve. A series of head-tail connected small line segments is generated as the final cutting tool path. This method has many shortcomings. The NURBS curve interpolation algorithm has a significant advantage ${ }^{[12]}$.

Because currently there has no open testing prototype in the lab, the NURBS curve interpolation algorithm and acceleration and deceleration control algorithm can not be verified through the actual parts cutting experiment, but the computer simulation can be used to analyze the efficiency and validity of the algorithm.

\section{Verifying approach}

(1) The simulation is conducted using software VERICUT of CNC processing simulation system.

(2) Enter the curve parameters and processing parameters to the NURBS curve interpolation software, the coordinates of each interpolation point location are generated.

(3) After NC program generated, using VERICUT software to simulate process parts and verify the correctness of the interpolation algorithm ${ }^{[3.4 .5]}$.

(4) The verification of acceleration and deceleration control algorithm is accomplished by the Origin graphic visualization and data analysis software.

\section{Verifying the correctness of the NURBS curve interpolation algorithm}

As Figure 1 shows a portion of the three-dimensional NURBS curve drawn by SOLIDWORKS software. The control points of the NURBS curve and the weighted factors are shown in Table 1. Interpolation parameters are: the interpolation cycle $\mathrm{Ts}=1 \mathrm{~ms}$, the feed rate $\mathrm{F}=20 \mathrm{~mm} / \mathrm{s}$, the maximum acceleration $\mathrm{a}_{\max }=100 \mathrm{~mm} / \mathrm{s}^{2}$, the limited chord error $\mathrm{ER}=1 \mu \mathrm{m}$. Node vector is $(0,0,0,0,0.06,0.1,0.14,0.22,0.25,0.30,0.34,0.43,0.49,0.53,0.56,0.64,0.71,0.78,0.83,0.88,1,1,1,1)$ 
Table 1 Data points and weights

\begin{tabular}{c|c|c|c|c|c|c|c|c|c|c|c|c|c|c|c|c|c|c|c|c}
\hline & 1 & 2 & 3 & 4 & 5 & 6 & 7 & 8 & 9 & 10 & 11 & 12 & 13 & 14 & 15 & 16 & 17 & 18 & 19 & 20 \\
\hline $\mathrm{X} / \mathrm{mm}$ & 0 & 1 & 4 & 6 & 7 & 7 & 4 & 3 & 4 & 13 & 21 & 26 & 29 & 22 & 14 & 4 & -3 & -5 & -3 & 0 \\
\hline $\mathrm{Y} / \mathrm{mm}$ & 0 & 0 & -3 & -7 & -11 & -20 & -18 & -14 & -10 & -6 & -5 & -6 & -9 & -18 & -22 & -24 & -22 & -16 & -6 & 0 \\
\hline $\mathrm{Z} / \mathrm{mm}$ & 0 & -1 & -7 & -11 & -16 & -22 & -19 & -14 & -9 & 0 & 3 & 4 & 4 & 1 & -1 & -3 & -4 & -2 & 0 & 0 \\
\hline $\mathrm{W}$ & 1 & 1 & 1 & 1 & 1 & 1 & 1 & 1 & 1 & 1 & 1 & 1 & 1 & 1 & 1 & 1 & 1 & 1 & 1 & 1 \\
\hline
\end{tabular}

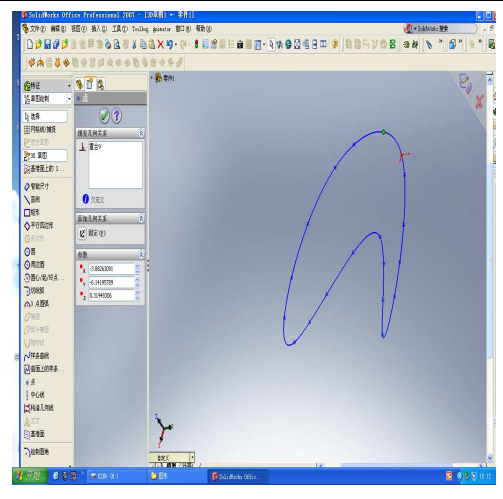

Fig.1 The NURBS example

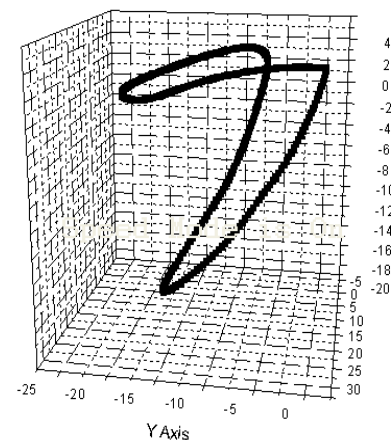

Fig. 2 Using Origin verified the NURBS example

Figure 2 is the generated interpolation location coordinates, which is the result of processing in the NURBS curve interpolation software and simulating by software Origin, which is the same as the curve drawn by SOLIDWORK, it verified the correctness of the NURBS curve interpolation algorithm. Figure 3 is the result of the first-order approximation interpolation algorithm. Figure 4 is the result of using the adaptive limit curvature and chord error algorithm.

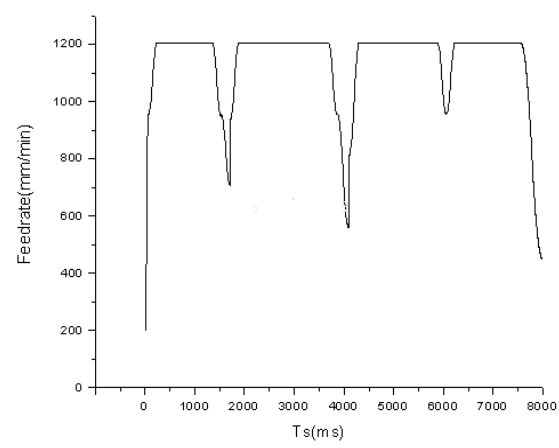

a. Feedrate graph

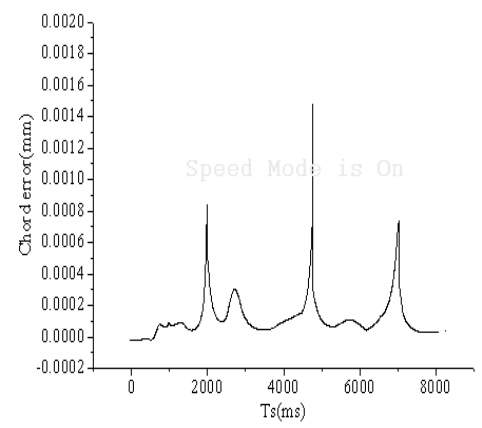

b. Contour error

Fig.3 Results of the first-order approximation interpolation algorithm

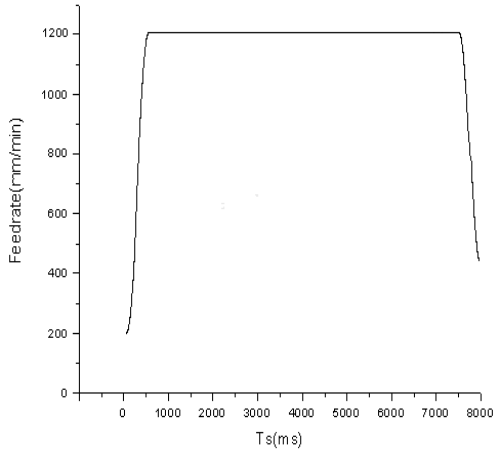

a. Feedrate graph

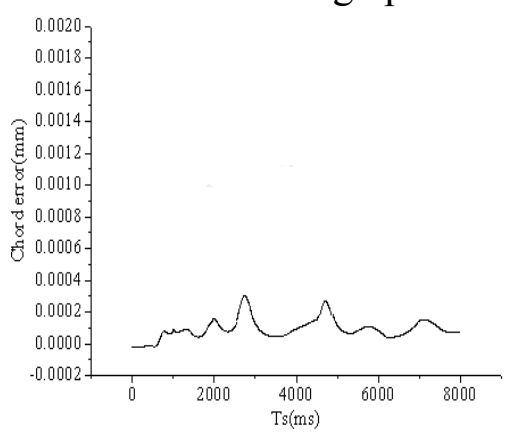

b. Contour error

Fig.4 Results of the interpolation algorithm with confined chord errors and max centripetal acceleration 
The simulation result shows:

(1) The smaller the interpolation curve curvature, the smaller the feed rate, the shorter the interpolation cycle, the smaller the error and the higher precision. From the actual measurement of the curvature can be seen: the four larger corresponding interpolation curve, but only three of curvature is greater than the maximum value $=0.25$.

(2) Without considering the Chord error and the maximum normal acceleration limits, the speed has no big change to reach the commanding feed rate to $(3000 \mathrm{~mm} / \mathrm{min})$, but at the large curvature of the angular contour the error apparently increased, to $1.5 \mu \mathrm{m}$, the speed does not decay.

(3) Although there are feed rate fluctuations at the three large curvature sharp corners of the curve $(450.4 \mathrm{~mm} / \mathrm{min}-1200 \mathrm{~mm} / \mathrm{min})$, but the contour error is significantly reduced (maximum $0.33 \mu \mathrm{m}$ ).

Add the command in the VERICUT simulation to process the component, it is shown in Figure 5.

\section{Feature comparison of all types of acceleration and deceleration algorithm}

Figure 5 is a portion of a cubic NURBS curve drawn by UG software. The control points and the weighted factors of the NURBS curve are shown in Table 2.

Table 2 Data points and weights

\begin{tabular}{c|c|c|c|c|c|c}
\hline & 1 & 2 & 3 & 4 & 5 & 6 \\
\hline $\mathrm{X} / \mathrm{mm}$ & 5 & -5 & -5 & 5 & 5 & -5 \\
\hline $\mathrm{Y} / \mathrm{mm}$ & 4 & 5 & 1 & -3 & -5 & -4 \\
\hline $\mathrm{Z} / \mathrm{mm}$ & 2 & 2.5 & 0.5 & -1.5 & -2.5 & -2 \\
\hline $\mathrm{W}$ & 1 & 1 & 1 & 1 & 1 & 1 \\
\hline
\end{tabular}

Node vector is: $(0,0,0,0,0.41,0.72,1,1,1,1)$

Interpolation parameters are following.

(1) The straight-line acceleration and deceleration control: the interpolation cycle $\mathrm{Ts}=1 \mathrm{~ms}$, the feed rate $\mathrm{F}=20 \mathrm{~mm} / \mathrm{s}$, the maximum acceleration $\mathrm{a}_{\max }=100 \mathrm{~mm} / \mathrm{s}^{2}$, the limited chord error $\mathrm{ER}=1 \mu \mathrm{m}$.

(2) The five S-phased acceleration and deceleration control: interpolation cycle $\mathrm{Ts}=1 \mathrm{~ms}$, the feed rate $F=20 \mathrm{~mm} / \mathrm{s}$, maximum acceleration $a_{\max }=100 \mathrm{~mm} / \mathrm{s}^{2}$, jerk $\mathrm{j}=500 \mathrm{~mm} / \mathrm{s}^{3}$, the limited chord error $\mathrm{ER}=1 \mu \mathrm{m}$, the initial speed $\mathrm{V}_{\mathrm{s}}=3 \mathrm{~mm} / \mathrm{s}$, the terminated velocity $\mathrm{V}_{\mathrm{e}}=8 \mathrm{~mm} / \mathrm{s}$.

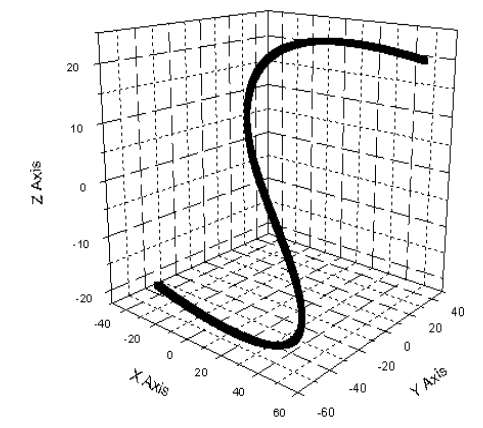

Fig. 5. The NURBS example

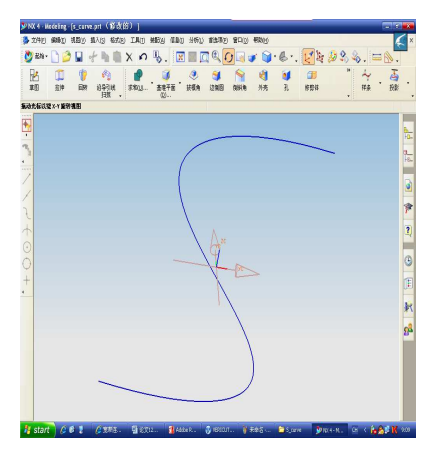

Fig. 6. Using Origin verified the NURBS example 


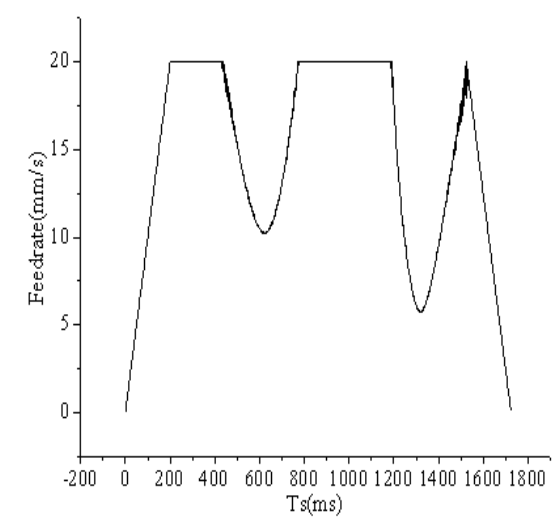

a. Feed rate graph

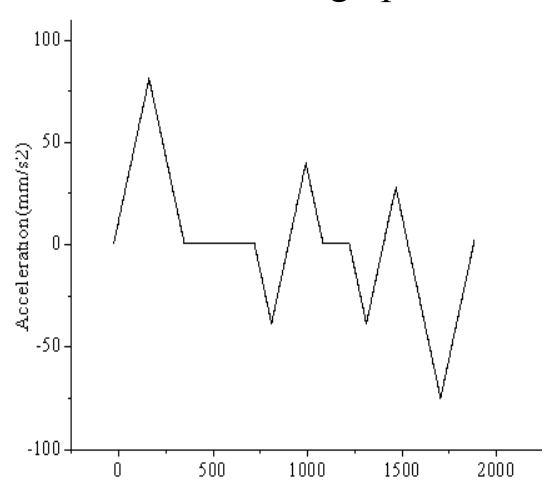

b. Acceleration graph

Fig.7 Line acceleration/deceleration control

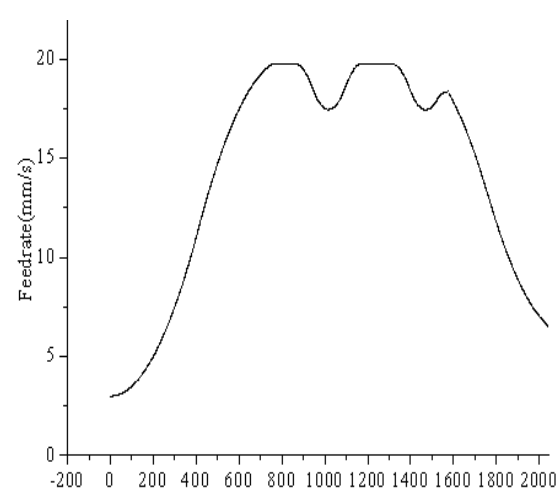

a. Feed rate graph

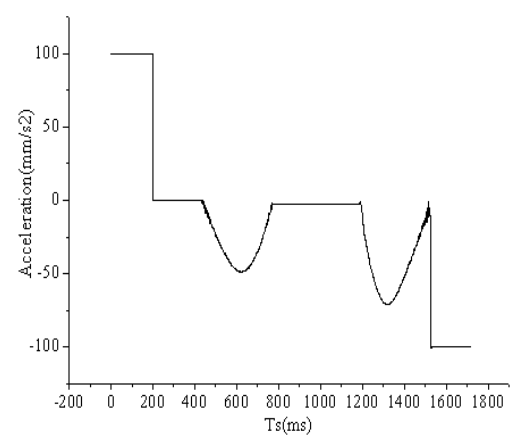

b. Acceleration graph

Fig.8 The five $\mathrm{S}$ - phased acceleration /deceleration control

Figure 6 is the result of processing in the NURBS curve interpolation software and simulating by software Origin, which is the same as the curve drawn by UG.

The simulation result shows (Figure 7 and Figure 8)

(1) Comparing to the straight-line acceleration and deceleration control method, the acceleration changes of the five S-phased curve acceleration and deceleration control method is more smooth, the speed curve is more flexible, but the movement time is longer $(2040 \mathrm{~ms}-1724 \mathrm{~ms}=316 \mathrm{~ms})$.

(2) During acceleration and deceleration process, the straight-line acceleration and deceleration control method exists four shocks at the four locations (in $1 \mathrm{~ms}, 200 \mathrm{~ms}, 1525 \mathrm{~ms}, 1724 \mathrm{~ms}$, amplitude up to $100 \mathrm{~mm} / \mathrm{s}^{2}$ ), which affects the cutting tool lasting life and processing quality; but the five S-phased acceleration deceleration control method in the whole acceleration and deceleration process has no impact, which greatly improves the processing accuracy.

(3) The straight-line acceleration and deceleration control method (433ms 772ms, $1186 \mathrm{~ms} \sim 1524 \mathrm{~ms}$ ) appeared acceleration oscillation (amplitude up to $14.82 \mathrm{~mm} / \mathrm{s}^{2}-7.66 \mathrm{~mm} / \mathrm{s}^{2}=7.16 \mathrm{~mm} / \mathrm{s}^{2}$ ), which affects the machining accuracy; and five S-phased curve acceleration and deceleration control method has smoother transition in this range (amplitude changes less than $0.5 \mathrm{~mm} / \mathrm{s}^{2}$ ), which is only $6.98 \%$ of the former.

(4) The straight-line acceleration and deceleration control method (433ms 772ms, $1186 \mathrm{~ms} \sim 1524 \mathrm{~ms}$ ) appeared the speed of oscillation (amplitude up to $20 \mathrm{~mm} / \mathrm{s}-15.85 \mathrm{~mm} / \mathrm{s}=4.15 \mathrm{~mm} / \mathrm{s}$ ), which affects the machining accuracy; and five S-shaped acceleration and deceleration control method has relatively smooth transition in this range (amplitude changes less than $0.3 \mathrm{~mm} / \mathrm{s}$ ), which is only $7.23 \%$ of the former.

The component processed with VERICUT simulation is shown in Figure 9. 


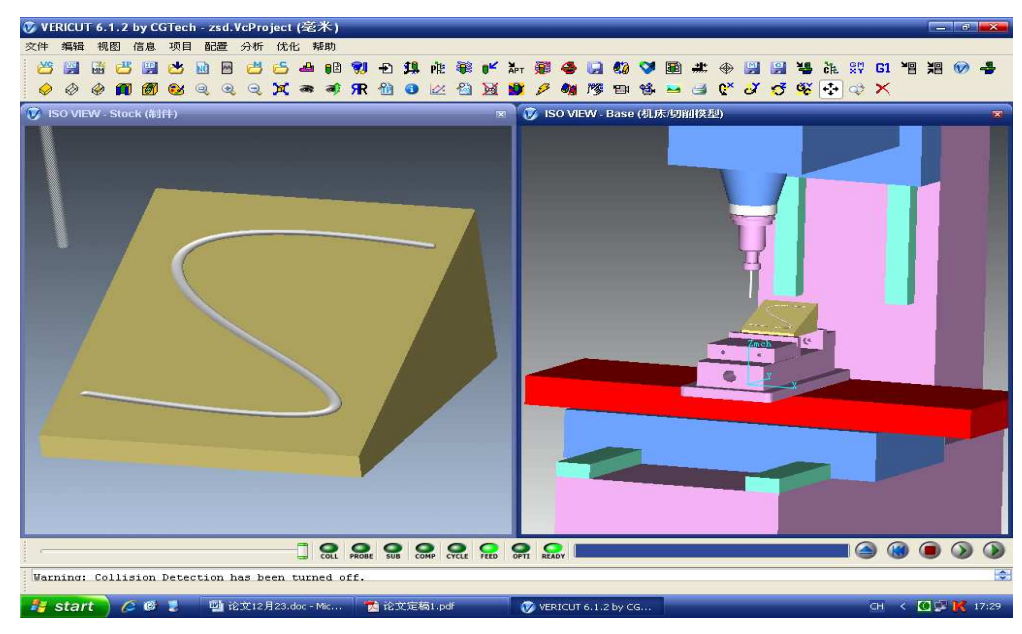

Fig.9 Part milled NURBS interpolation

\section{Summary}

The correctness of the NURBS curve interpolation algorithm is verified with the simulation. Then, an important conclusion can be obtained: the five S-shaped curve acceleration and deceleration control method has good flexibility, it can be used to prevent impact when the speed has sudden changes, out of step, over run or oscillation, ensured smooth movement.

\section{References}

[1] Daniel CH Yang and Tom Kong.parametric interpolator versus linear interpolator for precision CNC machining [J].Computer Aided Design.1994,26(3):225-234

[2] S Bedi,I Ali, and N Quan.Advanced interpolation techniques for NC machines[A].Transactions of the ASME Journal of Engineering for Industry.1993,115:329-336

[3] M Shpitalni,Y koren,and C C Lo.Realtime curve interpolators[J].Computer Aided Design. 1994, 26(11):832-838

[4] M Y Cheng,M C Tsai,and J C Kou.Real-time NURBS commang generators for CNC servo controllers[J].International Journal of Machine Tools and Manufacture. 2002,42:801-387

[5] S.-S. Yeh, R.-L. Hsu. Adaptive-feedrate interpolation for parametric curves with a confined chord error. Computer Aided Design 2002,34:229-237 